\title{
768 SYSTEMIC DELIVERY OF A TUMOR-TARGETED TLR9 AGONIST CONJUGATE TRANSFORMS THE TUMOR IMMUNE LANDSCAPE AND INDUCES TUMOR REGRESSION IN MICE
}

Caitlyn Miller Candidate*, Idit Sagiv-Barfi, Patrick Neuhoefer, Debra Czerwinski, Steven Artandi, Carolyn Bertozzi, Ronald Levy, Jennifer Cochran. Stanford University, Stanford, CA, United States

Background Tumor-localized delivery of Toll-like receptor (TLR) agonists is a promising strategy to promote immune activation within the tumor microenvironment (TME) to overcome tumor immunosuppression and induce anti-tumor immune responses. To enable localization to multiple tumor sites following systemic administration, we developed a fullysynthetic tumor-targeting TLR9 agonist and demonstrate its potential to transform the tumor immune microenvironment for effective tumor regression in mice.

Methods An engineered synthetic peptide (PIP) that binds to multiple integrin receptors overexpressed in many solid tumors was chemically conjugated to a synthetic CpG oligonucleotide (TLR9 agonist), thereby generating a tumor-targeting immunestimulant referred to as PIP-CpG. To facilitate clinical translation, PIP-CpG is cross-reactive between mouse, non-human primate, and human. Therapeutic studies were conducted in immune-competent mice bearing breast or pancreatic tumors to evaluate the efficacy of intravenously (IV)-injected PIP-CpG compared to IV-injected unmodified $\mathrm{CpG}$ or vehicle (PBS). We then performed mechanistic studies to evaluate the immune response elicited by PIP-CpG therapy.

Results Intravenous dosing of PIP-CpG led to tumor regression and prolonged survival, and in some cases cures, relative to vehicle or unmodified $\mathrm{CpG}$ therapy in both murine breast (4T1) and pancreatic cancer (KPC-G2) models. This tumor regression was dependent on $\mathrm{T}$ cells as $\mathrm{T}$ cell depletion resulted in loss of therapeutic response. To study the effect of systemic therapy on the cellular landscape in the TME, we analyzed 4T1 breast tumors by flow cytometry. We found that vehicle and $\mathrm{CpG}$ IV-dosed mice had immunosuppressive TMEs comprised mostly of myeloid-derived suppressor cells (MDSCs; $43-68 \%$ of live cells) with minimal infiltrating $\mathrm{T}$ cells and $\mathrm{B}$ cells $(5-16 \%$ of live cells). In contrast, the TME of PIP-CpG treated mice was transformed into a lymphocyte-rich "hot" tumor phenotype with massive infiltration by $\mathrm{T}$ cells and $\mathrm{B}$ cells (92-95\% of live cells) and plummeting levels of MDSCs (down to $\sim 1 \%$ ). In addition, tumor-specific effector CD8 $+\mathrm{T}$ cells were generated in response to PIP-CpG therapy, but not CpG dosed IV, indicating that PIP-CpG therapy transforms the TME and elicits a $\mathrm{T}$ cell-mediated tumor-specific immune response. Furthermore, PIP-CpG was effective for treating MMTV-PyMT transgenic mice, which spontaneously develop multiple breast tumors. Murine toxicity studies indicated that effects of PIP-CpG were similar to CpG dosed IV or intratumorally, which have been well-tolerated in human clinical trials.

Conclusions Tumor-directed systemic delivery of a TLR9 agonist transforms the TME via activated $\mathrm{B}$ and $\mathrm{T}$ cells and is promising for further development in patients with solid tumors.

Ethics Approval All mouse experiments were performed in accordance with protocols approved by the Stanford Administrative Panel on Laboratory Animal Care.

http://dx.doi.org/10.1136/jitc-2021-SITC2021.768 